\title{
Fundamental Oscillation Periods of the Interlayer Exchange Coupling beyond the RKKY Approximation
}

\author{
M. S. Ferreira $\dagger$, J. d'Albuquerque e Castrot $\ddagger$, D. M. Edwards $\dagger$ and J. Mathon $\ddagger$ \\ $\dagger$ Department of Mathematics, Imperial College, London, SW7 2BZ, UK \\ $\ddagger$ City University, London, EC1V OHB, UK
}

(July 12, 2021)

\begin{abstract}
A general method for obtaining the oscillation periods of the interlayer exchange coupling is presented. It is shown that it is possible for the coupling to oscillate with additional periods beyond the ones predicted by the RKKY theory. The relation between the oscillation periods and the spacer Fermi surface is clarified, showing that non-RKKY periods do not bear a direct correspondence with the Fermi surface. The interesting case of a FCC(110) structure is investigated, unmistakably proving the existence and relevance of non-RKKY oscillations. The general conditions for the occurrence of nonRKKY oscillations are also presented.
\end{abstract}

PACS numbers: 75.50.Fr, 75.30.Et, 75.50.Rr

Typeset using REVTEX

*Permanent address: Instituto de Física, Universidade Federal Fluminense, Niterói, Rio de Janeiro, 24001-970, Brazil 


\section{INTRODUCTION}

Oscillatory exchange coupling between metallic magnetic layers across a non-magnetic spacer has been intensively studied over the last few years. One of the main issues in this field has been the determination of the oscillation periods of the coupling as a function of the spacer thickness. The physical mechanisms proposed for explaining the so-called oscillatory interlayer coupling include the quantum well theory (QWT) of Edwards et al. [1, 2, [3] and an extension of the Ruderman-Kittel-Kasuya-Yosida (RKKY) theory adapted to the multilayer geometry due to Bruno and Chappert [4, [5].

Both theoretical approaches seem to agree that the origin of the oscillation periods is in the structure of the spacer Fermi surface (FS). In fact, in the RKKY theory the periods are associated with wave vectors directly obtained from the FS. More specifically, they are given by vectors perpendicular to the layer planes which span two points of the FS with antiparallel Fermi velocities [4,5]. There has been a general belief that all oscillation periods are given by the RKKY theory and therefore that such a simple picture is always valid. Indeed, it has been shown that in certain simple models [1, 2, 3] the periods predicted by the QWT coincide with the RKKY ones and are given by the extremal dimensions of the spacer FS in the direction perpendicular to the layers. In another case [10], where the lattice lacks reflection symmetry about the layer planes, the correspondence between the quantum well and the RKKY periods is rather subtle but still obtains. In the models mentioned above, harmonics of the RKKY periods are obtained but no new fundamental periods. Furthermore, d'Albuquerque e Castro et al. [6] showed analytically for a very general model that RKKY theory holds in the limit of small exchange splitting in the ferromagnetic material.

However, using a more general approach [6, 15] based on the existence of quantum well states [1, 2, 3], fundamental periods not predicted by RKKY theory have been recently obtained for the particular case of a FCC lattice along the (110) direction [7]. In that communication, within the single-band tight-binding model with nearest neighbour interaction, it is shown that in addition to the RKKY predictions, a number of distinct periods is involved in 
the coupling. Those new periods arise from non-negligible higher order terms which RKKY, as a second order perturbative approach, does not account for. The resemblance of the FS considered with those of the noble metals suggests possible real effects in systems having these materials as spacers. Moreover, the occurrence of non-RKKY behaviour within the simplest single-band model indicates that it should also happen in the more elaborate multiband case. In fact, non-magnetic transition metals with their multi-sheet Fermi surfaces are suitable candidates for presenting non-RKKY periods. Furthermore, in most cases investigated so far, each oscillation period comes from distinct $\mathbf{q}_{\|}$-points of the 2-dimensional Brillouin zone (BZ). Even when multi-periodic oscillations occur, one value of $\mathbf{q}_{\|}$yields only one period. The existence of more than one period for a single value of $\mathbf{q}_{\|}$, which may arise in a number of cases, has not been thoroughly investigated and the combination of such periods may lead to new features of the oscillations. For the reasons above, a general understanding of the mechanisms determining all oscillation periods is needed.

The purpose of this work is to generalize the method previously used to calculate the oscillation periods of the interlayer coupling. The relation between the periods and the spacer FS is clarified and in addition to the determination of the usual periods, the general conditions for occurrence of non-RKKY behaviour are also established. Furthermore, here we present a rather detailed set of results in order to illustrate better the different stages of the calculations. The sequence of the paper is as follows. In section \ we present a general method for obtaining the oscillation periods of the interlayer coupling, where in addition to the determination of the usual periods, the existence of non-RKKY behaviour is proved to be possible. Similarly to previous theoretical approaches [4, 5, 1, 3, 16, an expression for the coupling in the asymptotic limit of large spacer thickness is derived. Here however, the occurrence of multi-periodicity for a single value of $\mathbf{q}_{\|}$becomes evident at the same time that the relation between the oscillation periods and the spacer FS is clarified. In section III, in order to illustrate the method, we look at the particular case of the FCC lattice along the (110) direction which unmistakably shows the relevance of terms beyond RKKY approximation. Finally, in the last section we conclude and present the general conditions 
for occurrence of non-RKKY oscillations.

\section{GENERAL METHOD}

Based on the formalism introduced by d'Albuquerque e Castro et al. [6], where the coupling is expressed in terms of the one-electron Green's functions of the multilayered system, we here present a general method for determining all the oscillation periods involved in the coupling.

For the sake of simplicity, we consider a system consisting of two parallel magnetic planes embedded in an infinite non-magnetic material. We label the two ferromagnetic planes 0 and $n$, so that the number of non-magnetic spacer planes is $n-1$. As far as the coupling as a function of the spacer thickness is concerned the number of magnetic planes does not affect the periods [8], influencing only phase and amplitude of the oscillations. Since the periods are our main concern, the restriction to two single magnetic planes does not pose any limitations on the results here obtained.

The expression for the coupling $\Delta \Omega(\pi)$, namely the difference in the thermodynamical potential $\Omega$ between the ferromagnetic and antiferromagnetic configurations, is given by [6]

$$
\Delta \Omega(\pi)=\frac{1}{\pi} \sum_{\mathbf{q}_{\|}} \int d \omega f(\omega) \mathcal{F}\left(\mathbf{q}_{\|}, \omega\right),
$$

where the function $\mathcal{F}$ is given by

$$
\mathcal{F}\left(\mathbf{q}_{\|}, \omega\right)=\operatorname{Im} \operatorname{tr} \ln \left\{1+4 G_{n 0}^{\uparrow}\left(\mathbf{q}_{\|}, \omega\right) V_{e x} G_{0 n}^{\downarrow}\left(\mathbf{q}_{\|}, \omega\right) V_{e x}\right\}
$$

In the equations above $G_{n 0}^{\sigma}\left(\mathbf{q}_{\|}, \omega\right)$ is the propagator between planes 0 and $n$ for electrons with spin $\sigma$ in the ferromagnetic configuration of the system, $f(\omega)$ is the Fermi function, $V_{e x}$ is a diagonal matrix in orbital indices representing the strength of the local exchange potential in the ferromagnetic layers, and the summation over $\mathbf{q}_{\|}$is restricted to the two-dimensional Brillouin zone (BZ). It is worth stressing that all matrices above are in orbital indices. We assume for simplicity that the non-magnetic part of the potential in the ferromagnetic planes 
is identical to the spacer on-site potential. This is not an essential assumption and can be lifted without major effects on the results. In fact, it has been shown that the presence of a spin-independent square well does not influence the periods of the interlayer coupling, affecting only the phase and amplitude of the oscillation [9]. As such, the exchange splitting of the magnetic material acts as two localized perturbations on the planes 0 and $n$ and in this situation the propagators $G$ can be written as a function of the bulk spacer propagators $g_{0 n}$ and $g_{n 0}$, i.e.,

$$
G_{n 0}^{\sigma}\left(\mathbf{q}_{\|}, \omega\right)=G^{\sigma}\left(g_{0 n}\left(\mathbf{q}_{\|}, \omega\right), g_{n 0}\left(\mathbf{q}_{\|}, \omega\right)\right)
$$

Within the particular case of the single-band model the explicit dependence of $G^{\sigma}$ upon the off-diagonal propagators $g_{0 n}$ and $g_{n 0}$ is, for each $\mathbf{q}_{\|}$and $\omega$, given by

$$
G_{n 0}^{\uparrow, \downarrow}= \pm \frac{\tau_{\uparrow, \downarrow} g_{n 0}\left(1-\tau_{\uparrow, \downarrow} g_{00}\right)}{V_{e x}\left(1-\tau_{\uparrow, \downarrow} g_{n 0} \tau_{\uparrow, \downarrow} g_{0 n}\right)},
$$

where $g_{\ell m}$ is a general matrix element of the bulk spacer Green's function and $\tau_{\uparrow, \downarrow}=$ $\pm V_{e x}\left(1 \pm V_{e x} g_{00}\right)^{-1}$. An analogous expression can be obtained for $G_{0 n}^{\uparrow, \downarrow}$. Note that whereas Eq.(3) involves matrices in orbital indices, all terms in Eq.(四) are scalars.

Returning to the general case and for reasons of future comparison, it is worth presenting the RKKY-limit expression for the bilinear coupling $J_{1}^{R K K Y}$ [6,9]. It is given by Eq.(1) with $\mathcal{F}$ replaced by

$$
\mathcal{F}_{R K K Y}\left(\mathbf{q}_{\|}, \omega\right)=\operatorname{Im} \operatorname{tr}\left\{2 V_{e x}^{2} g_{n 0}\left(\mathbf{q}_{\|}, \omega\right) g_{0 n}\left(\mathbf{q}_{\|}, \omega\right)\right\}
$$

We recall that the expansion of $\Delta \Omega(\theta)$ in powers of $\cos (\theta)$, being $\theta$ the angle between the magnetization vectors in the magnetic planes, defines the bilinear and biquadratic couplings $J_{1}$ and $J_{2}$, respectively [11]. Moreover, it is found that higher order terms than $\cos ^{2}(\theta)$ are negligible so that $J_{1}=\Delta \Omega(\pi) / 2[9]$.

It is clear from Eqs.(2), (3) and (5) that in either case the spacer thickness dependence of the interlayer coupling is entirely determined by the off-diagonal propagators $g_{0 n}$ and $g_{n 0}$. The investigation of the oscillation periods thus requires an analysis of $g_{n 0}$ as a function of $n$. 


\section{A. Calculation of the 1-dimensional propagators}

For fixed values of $\mathbf{q}_{\|}$, the Green's functions $g$ are equivalent to 1-dimensional propagators with $\mathbf{q}_{\|}$-dependent hoppings and in-plane energies. We recall that $g$ is a matrix over orbital indices and therefore a general matrix element $g_{\ell m}^{\mu \nu}$ represents the propagation of an electron with orbital $\mu$ from plane $\ell$ into an orbital $\nu$ at plane $m$. It is given by

$$
g_{\ell m}^{\mu \nu}\left(\mathbf{q}_{\|}, \omega\right)=\left(\frac{d}{2 \pi}\right) \sum_{s} \int_{-\frac{\pi}{d}}^{\frac{\pi}{d}} d q_{\perp} \frac{a_{s \mu}^{*}\left(q_{\perp}\right) a_{s \nu}\left(q_{\perp}\right) e^{-i q_{\perp}(\ell-m) d}}{\omega-E_{s}\left(\mathbf{q}_{\|}, q_{\perp}\right)+i 0^{+}},
$$

where $q_{\perp}$ is the wave vector perpendicular to the layers, $d$ is the interplane distance, $E_{s}\left(\mathbf{q}_{\|}, q_{\perp}\right)$ describes the bulk spacer band structure, being $s$ the band index, and $a_{s \mu}\left(q_{\perp}\right) \equiv$ $\left\langle q_{\perp} s \mid q_{\perp} \mu\right\rangle$ is the projection of the eigenstate $s$ into the orbital $\mu$ for a given $q_{\perp}$.

The integral in Eq.(6) can be evaluated for $\ell<m$ by simply extending $q_{\perp}$ to the complex plane and changing the contour integration from a straight line on the real axis to the boundaries of a semi-infinite rectangle in the upper-half plane whose base lies on the real axis between $-\pi / d$ and $\pi / d$. For the case $\ell>m$, the extension is to a rectangle in the lower-half plane. In either case the integrand vanishes as $\left|\operatorname{Im} q_{\perp}\right| \rightarrow \infty$ and because $q_{\perp}=-\frac{\pi}{d}+i y$ and $q_{\perp}=\frac{\pi}{d}+i y$ are equivalent wave vectors, the integrals along the vertical sides of the rectangle cancel each other, simplifying the problem to the calculation of the residues associated with the poles of the integrand inside the closed contour. The poles are in turn given by the values of $q_{\perp}$ satisfying the condition $\omega^{+}-E_{s}\left(\mathbf{q}_{\|}, q_{\perp}\right)=0$, where $\omega^{+}=\omega+i 0^{+}$.

The poles are clearly dependent on the band structure. Due to the in-plane symmetry of the system, the general tight-binding Hamiltonian of the homogeneous system is written as

$$
H\left(\mathbf{q}_{\|}, q_{\perp}\right)=\sum_{r r^{\prime}} H_{r r^{\prime}}\left(\mathbf{q}_{\|}\right) e^{i q_{\perp}\left(r-r^{\prime}\right) d}
$$

where $H_{r r^{\prime}}\left(\mathbf{q}_{\|}\right)$are operators describing the electron hopping between planes $r$ and $r^{\prime}$. The upper limit of the sum depends on the maximum number of planes $\bar{r}$ connected through the electron hoppings as well as on the lattice structure. As we shall see, in the FCC lattice 
along the (110) direction for instance, even with only hoppings between nearest atoms being included, electrons may hop between nearest and next nearest planes. For a fixed value of $\omega^{+}$, the wave vectors $q_{\perp}$ satisfying the equation $\omega^{+}-E_{s}\left(\mathbf{q}_{\|}, q_{\perp}\right)=0$ are the solutions of a polynomial equation in $e^{i q_{\perp} d}$, i.e.,

$$
\sum_{r}^{\bar{r}} \Upsilon_{r}\left(\mathbf{q}_{\|}\right) e^{i q_{\perp} r d}=0
$$

where $\Upsilon_{r}\left(\mathbf{q}_{\|}\right)$is a function of the $\mathbf{q}_{\|}$-dependent hoppings and in-plane energies. Note that the solutions of the equation above are exponentials $e^{i q_{\perp} d}$ and not wave vectors $q_{\perp}$ themselves. We label the poles $q_{j}^{s}$, where $j$ numbers the poles of a given band index $s$. Since $E_{s}\left(q_{\perp}\right)=$ $E_{s}\left(-q_{\perp}\right)$ poles appear in pairs and may be real or complex. We see from a simple Taylor expansion of $\omega-E_{s}\left(\mathbf{q}_{\|}, q_{\perp}\right)$ around the real poles that, for a given pair, which pole contributes to the integral depends on the sign of the derivative $\left(\partial E_{s} / \partial q_{\perp}\right)_{q_{j}^{s}}$. For a given band $s$ which crosses the energy $w$ twice, yielding two pairs of real poles, the contributory poles $q_{1}^{s}$ and $q_{2}^{s}$ have different signs. In other words, when $q_{1}^{s}$ is in the range $[0, \pi], q_{2}^{s}$ is in the range $[-\pi, 0]$ or vice versa.

It is interesting to look at the physical significance of the real poles. Being obtained from the band structure $E_{s}\left(\mathbf{q}_{\|}, q_{\perp}\right)$, the poles $q_{j}^{s}$ are just the wave vectors with which electrons of energy $\omega$ having in-plane energies $\epsilon\left(\mathbf{q}_{\|}\right)$and hoppings $t\left(\mathbf{q}_{\|}\right)$propagate across the spacer. Thus, for $\omega=E_{F}$, where $E_{F}$ is the Fermi energy, the real poles are just the perpendicular coordinates of the FS for a fixed value of $\mathbf{q}_{\|}$. As discussed later, complex poles contribute significantly only in the case of thin spacers.

By adding the residues associated with the contributory poles we obtain an analytical expression for $g_{\ell m}$, given by

$$
g_{\ell m}\left(\mathbf{q}_{\|}, \omega\right)=\sum_{s} \sum_{j} A_{s j} e^{-i q_{j}^{s}(\ell-m) d}
$$

where the matrix elements of the matrix $A_{s j}$ are

$$
A_{s j}^{\mu \nu}\left(\mathbf{q}_{\|}, \omega\right)=-i d a_{s \mu}^{*}\left(q_{j}^{s}\right) a_{s \nu}\left(q_{j}^{s}\right)\left\{\left[\frac{\partial E_{s}\left(\mathbf{q}_{\|}, q_{\perp}\right)}{\partial q_{\perp}}\right]_{q_{\perp}=q_{j}^{s}}\right\}^{-1} .
$$


The coefficients $A_{s j}^{\mu \nu}$ depend neither on $\ell$ nor on $m$ and the only dependence on the distance between the planes is inside the argument of the exponentials. In addition, the exponentials are independent of the orbital indices, which means that all matrix elements oscillate with the same periods. Note that in calculating Eq.(10) we have assumed that the coefficients $a_{s \mu}\left(q_{\perp}\right)$ are not ill-behaved, that is, they have neither singularities nor branch points inside the integration contour. In this way, we just need to evaluate the coefficients at the poles $q_{j}^{s}$

Nevertheless, in a general case the coefficients $a_{s \mu}\left(q_{\perp}\right)$ are not well behaved. Although they do not have singularities like in the denominator of Eq.(6), branch points and cuts do exist. The contributions from the branch points must be taken into account otherwise a simple summation of the residues will not give the correct result of the integration. This is a very important point that, if overlooked, may lead to erroneous results. In fact, in a paper by Bruno [17] where the coupling is expressed in terms of transmission and reflection coefficients of electrons across quantum barriers, a similar integral to that in Eq.(6) arises. In that communication no particular attention was given to the analytic properties of the coefficients $a_{s \mu}\left(q_{\perp}\right)$ and the result above was erroneously concluded to be exact for arbitrary values of $\ell$ and $m$. It is important to stress that Eqs.(19) and (10) are not correct in general. The equations above become exact for all $\ell$ and $m$ only within the single-band model, where there are no coefficients introducing branch points. This is clearly seen in Fig.11 where the real and imaginary parts of the function $g_{05}(\omega)$ of a linear chain with hoppings up to four nearest neighbours within the single-band model are displayed. The parameters used were $\epsilon=0, t_{1}=-1 / 2, t_{2}=-1 / 2, t_{3}=1 / 5$ and $t_{4}=1 / 10$, where $\epsilon$ is the in-plane energy and $t_{\ell}$ are the hoppings to the $\ell$-th neighbour planes. The analytical results following Eq.(9), represented by the lines, are in absolute agreement with the brute-force calculation of Eq.(同), represented by the symbols. In the general case there are also contributions to the $q_{\perp}$ integral arising from branch cuts associated with the coefficients $a_{s \mu}\left(q_{\perp}\right)$. Since in general these cuts do not intersect the real axis, the factor $e^{i q_{\perp}(\ell-m) d}$ in the integrand of Eq.(6) ensures that the contribution is negligible for large $|\ell-m|$. Thus, failure to treat the branch cuts correctly 
does not introduce errors in the asymptotic limit of large $|\ell-m|$.

It is clear that $g_{\ell m}$ is an oscillatory function of the distance between the planes and it oscillates with different periods, each one associated with its respective wave vector $q_{j}^{s}$. In addition, the combination of exponentials allows a quasi-periodic behaviour of $g_{\ell m}$ because, in general, the wave vectors $q_{j}^{s}$ are incommensurate. This fact, as we shall see, may have a striking effect on the oscillatory coupling. Another point to be mentioned is the possibility of evaluating $g_{\ell m}$ in Eq.(9) for non-integer values of $\ell$ and $m$, which allows the evaluation of the coupling for continuous values of spacer thickness.

We now mention the case of complex poles. Since they have non-zero imaginary parts, their contribution for large separation between the planes is again negligible. Thus only real wave vectors contribute to the oscillations for large $|\ell-m|$. Bearing in mind that the principal contribution to the coupling comes from the energy region around the Fermi level [1, $2,3,3,[16]$, it is clear that at least one real wave vector will be involved in the expression for $g_{\ell m}$. Thus the sum over the band index $s$ in Eq.(9) must be only over the bands which cross the Fermi level.

\section{B. Multiple Fourier expansion}

Having investigated the function $g_{\ell m}$ as a function of the distance between the planes, we recall that $\mathcal{F}$ is expected to oscillate with the same periods. The obvious way of representing the function $\mathcal{F}$ is through a simple Fourier expansion, which nevertheless cannot be done due to the quasi-periodicity of $g$. In other words, because the wave vectors $q_{j}^{s}$ are in general incommensurate, $g_{0 n}$ and $g_{n 0}$ may have an overall non-periodic behaviour. To overcome this problem we make use of the multiple Fourier series, recently used to deal with the problem of magnetic thickness dependence of the interlayer coupling [8]. The procedure consists in replacing the spacer thickness $n$ multiplying the wave vectors $q_{j}^{s}$ in each exponential with fictitious spacer thicknesses $n_{j}^{s}$, such that Eq.(9) becomes 


$$
\bar{g}\left(n_{1}^{1}, \ldots, n_{j}^{s}, \ldots\right)=\sum_{s} \sum_{j} A_{s j} e^{-i q_{j}^{s} n_{j}^{s} d}
$$

The new function $\bar{g}$ is now periodic in each variable $n_{j}^{s}$ separately, and so is its corresponding $\overline{\mathcal{F}}$. Bearing in mind that $\bar{g}$ does not correspond to the physically real case, which is reproduced by making $n_{j}^{s}=n$ for every $j$ and $s$, we evaluate the Fourier series for each variable $n_{j}^{s}$ separately, eventually making them all equal $n . \mathcal{F}$ then becomes

$$
\mathcal{F}=\sum_{\left(m_{1}^{1}, \ldots, m_{j}^{s}, \ldots\right)} C_{\left(m_{1}^{1}, \ldots, m_{j}^{s}, \ldots\right)} e^{i n d \sum_{s j}\left(m_{j}^{s} q_{j}^{s}\right)}
$$

where $m_{j}^{s}$ are integers, $C_{\left(m_{1}^{1}, \ldots, m_{j}^{s}, \ldots\right)}$ are the Fourier coefficients given by

$$
C_{\left(m_{1}^{1}, \ldots, m_{j}^{s}, \ldots\right)}=\left(\frac{1}{\Lambda_{1}^{1} \ldots \Lambda_{j}^{s} \ldots}\right) \int_{0}^{\Lambda_{1}^{1}} d n_{1}^{1} \ldots \int_{0}^{\Lambda_{j}^{s}} d n_{j}^{s} \ldots \overline{\mathcal{F}}\left(n_{1}^{1}, \ldots, n_{j}^{s}, \ldots\right) e^{i d \sum_{s j}\left(m_{j}^{s} q_{j}^{s} n_{j}^{s}\right)}
$$

and $\Lambda_{j}^{s}=2 \pi /\left|q_{j}^{s}\right|$ are the periods associated with the wave vectors $q_{j}^{s}$. The number of indices involved in the Fourier coefficients is the same as the number of poles because each $q_{j}^{s}$ has been associated with a fictitious spacer thickness $n_{j}^{s}$. In spite of the somewhat congested notation, in practice there are only a few indices in the coefficients due to the restricted number of real solutions crossing the Fermi level. In fact, within the single-band model the calculation of the Fourier coefficients can be extremely simplified, as shown in appendix A. The analysis of such a simplified model, although primarily illustrative, reflects some properties common to the more general case. Two of these properties are $C_{-m_{1},-m_{2}}=C_{m_{1}, m_{2}}^{*}$ and $C_{m_{1}, m_{2}}=0$ for odd values of $m_{1}+m_{2}$.

\section{RKKY-Coefficients}

We now focus on the RKKY-limit expression for the coupling. This is the limit of small ferromagnetic exchange splitting. It is clear from Eq.(5) that unlike the coupling $\Delta \Omega$, the RKKY expression involves neither logarithmic functions nor combinations of $G_{n 0}^{\sigma}$ and $G_{0 n}^{\sigma}$.

In fact, $\mathcal{F}_{R K K Y}$ simply contains a single product of the propagators $g_{n 0}$ and $g_{0 n}$. Bearing Eq.(5) in mind we define $\mathcal{F}_{R K K Y} \equiv\left[\mathcal{F}_{R K K Y}^{\prime}-\left(\mathcal{F}_{R K K Y}^{\prime}\right)^{*}\right] / 2 i$ and from the expression for an arbitrary element $g_{\ell m}$ in Eq.(9) we write 


$$
\mathcal{F}_{R K K Y}^{\prime}=2 V_{e x}^{2} \sum_{\mu \nu} \sum_{s, s^{\prime}} \sum_{j, j^{\prime}} A_{s j}^{\mu \nu} A_{s^{\prime} j^{\prime}}^{\nu \mu} e^{i\left(q_{j}^{s}+q_{j^{\prime}}^{s^{\prime}}\right) n d} .
$$

Clearly, the above equation shows that the integrand $\mathcal{F}_{R K K Y}$ can be manipulated and put in a Fourier expansion form, similarly to $\mathcal{F}$ in Eq.(12). However, the main difference is that in this case the number of terms involved in the expansion is limited, related to the maximum number of poles in Eq.(9), whereas the sum in Eq.(12) runs over an infinite number of terms. Such difference is due to the fact that the RKKY-limit expression results from a perturbative approach corresponding to the lowest order term of an expansion. All the terms included in $\mathcal{F}_{R K K Y}$ are also included in the full expression $\mathcal{F}$. The higher order terms correspond to oscillations that are either harmonics of the fundamental RKKY behaviour or effectively new oscillation periods. This point confirms the RKKY theory as a limit of the more general approach used here. We also point out that in this case, the argument of

the exponentials has the form $\left(m_{j}^{s} q_{j}^{s}+m_{j^{\prime}}^{s^{\prime}} q_{j^{\prime}}^{s^{\prime}}\right)$, involving only two wave vectors and with the constraint $\left|m_{j}^{s}+m_{j^{\prime}}^{s^{\prime}}\right|=2$, being $\left|m_{j}^{s}\right| \leq 2$ and $\left|m_{j^{\prime}}^{s^{\prime}}\right| \leq 2$. This is a consequence of $\mathcal{F}_{R K K Y}$ being the product of only two propagators, namely, $g_{n 0}$ and $g_{0 n}$. In the more general case of $\mathcal{F}$, the infinite possibilities of combining the mentioned propagators does not pose such a restriction.

At this point we raise the question of whether the RKKY periods of the interlayer coupling remain valid as the exchange splitting of the ferromagnetic layers, associated with $V_{e x}$, is increased. The question can be reformulated as whether the higher order terms in $\mathcal{F}$, apart from the RKKY harmonics, do contribute to the coupling. The answer to this question is in the evaluation of the integrals in Eq.(1).

\section{Stationary phase approximation}

Having written $\mathcal{F}$ (and $\mathcal{F}_{R K K Y}$ ) in terms of a Fourier expansion, we can now make use of the stationary phase method [1, 2, 3, 3, 16], which yields an analytical expression for the coupling, asymptotically exact for thick spacers $(n>>1)$.

Inserting Eq.(12) into (1), we have 


$$
\Delta \Omega(\pi)=\frac{1}{\pi} \sum_{\left(m_{1}^{1}, \ldots, m_{j}^{s}, \ldots\right)} \sum_{\mathbf{q}_{\|}} \int d \omega f(\omega) C_{\left(m_{1}^{1}, \ldots, m_{j}^{s}, \ldots\right)} e^{i n d \sum_{s j}\left(m_{j}^{s} q_{j}^{s}\right)} .
$$

We recall that the coefficients $C_{\left(m_{1}^{1}, \ldots, m_{j}^{s}, \ldots\right)}$ and the wave vectors $q_{j}^{s}$ depend on both integration variables $\mathbf{q}_{\|}$and $\omega$. The stationary phase method shows that in the asymptotic limit of large spacer thickness the only contributions to the integral over $\mathbf{q}_{\|}$arise from the neihbourhood of points which make the arguments of the exponentials stationary. We call these points $\mathbf{q}_{\|}^{\alpha}$, where the superscript $\alpha$ labels the different vectors. Furthermore, the integral over $\omega$ is also non-negligible only around the Fermi energy $E_{F}$. Hence, the wave vectors that effectively contribute to the coupling must satisfy the following condition,

$$
\sum_{s j}\left[m_{j}^{s} \nabla_{\|} q_{j}^{s}\left(\mathbf{q}_{\|}, E_{F}\right)\right]=0
$$

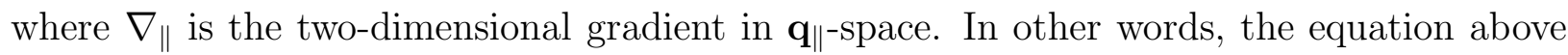
gives the necessary conditions for a constructive interference between the electrons across the spacer. The otherwise destructive interference does not contribute to the coupling. Eq.(16) simply selects the effective periods with which the coupling oscillates.

Note that for $\omega=E_{F}$, each wave vector $q_{j}^{s}\left(\mathbf{q}_{\|}, E_{F}\right)$ represents a surface in the reciprocal space and when put together, the surfaces $q_{j}^{s}$ reproduce the spacer Fermi surface. Following this argument and bearing in mind that non-real values of $q_{j}^{s}$ strongly damp the oscillations, we stress that only real solutions must be taken into account. The possible stationary solutions of Eq.(16) reflect the lattice structure and are usually located at points of high symmetry, even though they may occur at general points of the 2-dimensional BZ.

In such limit we obtain an analytical expression for the coupling given by [1, 3 , 8 ]

$$
\begin{gathered}
\Delta \Omega(\pi)=\sum_{\alpha} \sum_{\left(m_{1}^{1}, \ldots, m_{j}^{s}, \ldots\right)} \mathcal{K}_{\left(m_{1}^{1}, \ldots, m_{j}^{s}, \ldots\right)}^{\alpha} e^{i n d \bar{q}}, \\
\mathcal{K}_{\left(m_{1}^{1}, \ldots, m_{j}^{s}, \ldots\right)}^{\alpha}=\left(\frac{2 \sqrt{2} d}{\pi \beta n}\right) \frac{\left.\sigma_{\left(m_{1}^{1}, \ldots, m_{j}^{s}, \ldots\right)}^{\alpha} C_{\left(m_{1}^{1}, \ldots, m_{j}^{s}, \ldots\right)}^{\alpha} \frac{\partial^{2} \bar{q}}{\partial q_{x}^{2}} \frac{\partial^{2} \bar{q}}{\partial q_{y}^{2}}\right|^{-1 / 2}}{\sinh \left[\left(\frac{\partial \bar{q}}{\partial \omega}\right)_{E_{F}} \frac{\pi n d}{\beta}\right]},
\end{gathered}
$$

where $\bar{q}=\sum_{s j}\left(m_{j}^{s} q_{j}^{s \alpha}\right), q_{j}^{s \alpha}=q_{j}^{s}\left(\mathbf{q}_{\|}^{\alpha}, E_{F}\right)$, and $\beta=1 / k_{B} T$. Here we neglect the energy dependence of the Fourier coefficients which in some cases 16 can be important. The factor 
$\sigma_{\left(m_{1}^{1}, \ldots, m_{j}^{s}, \ldots\right)}^{\alpha}$ takes the value $i$ for a minimum, $-i$ for a maximum and 1 for a saddle point of the surface $\bar{q}\left(q_{x}, q_{y}\right)$. The sum over $\alpha$ regards all different stationary solutions and the inner sum over $\left(m_{1}^{1}, \ldots, m_{j}^{s}, \ldots\right)$ runs over the values allowed by the stationary phase condition. The coupling oscillates with periods $\lambda_{\left(m_{1}^{1}, \ldots, m_{j}^{s}, \ldots\right)}^{\alpha}=2 \pi /\left|\sum_{s j}\left(m_{j}^{s} q_{j}^{s \alpha}\right)\right|$ and their weights depend on geometric factors as well as on the Fourier coefficients.

An important issue is the relationship between the Fermi surface and the wave vectors that effectively contribute to the coupling. The lowest order combinations amongst the possible wave vectors $q_{j}^{s}\left(\mathbf{q}_{\|}, E_{F}\right)$ bear a direct correspondence with the FS. Indeed, this correspondence agrees with the RKKY geometrical picture for selecting the oscillation periods of the interlayer coupling. However, in the general case of a higher order combination of wave vectors the RRKY relation no longer holds, and the effective wave vectors are not directly related to the spacer FS. This is a very important result which shows that the geometrical criteria used for selecting the oscillation periods of the interlayer coupling are incomplete. Even though the wave vectors $q_{j}^{s}$ are directly related to the FS, the general wave vectors $\sum_{s j}\left(m_{j}^{s} q_{j}^{s}\right)$ are not. Furthermore, note that if $g_{n 0}\left(\mathbf{q}_{\|}^{\alpha}, E_{F}\right)$ has only one oscillation period, the full expansion in Eq. (17) contains merely harmonics of the fundamental RKKY periods. In other words, if the FS is cut in a single point by the line drawn from the origin perpendicular to the layers for a fixed $\mathbf{q}_{\|}$, the RKKY periods and its harmonics are the only ones present in the coupling. In fact, it explains the agreement between the periods predicted by the RKKY and QWT in the models previously mentioned [1, 2, 3, 10]. For a multi-periodic $g_{n 0}\left(\mathbf{q}_{\|}, E_{F}\right)$ though, where the FS is intersected in more than one point corresponding to incommensurate wave vectors, the higher order combinations of the wave vectors correspond neither to a RKKY period nor to any of its harmonics, but to fundamentally new periods.

Multi-periodicity of the off-diagonal propagator is a necessary but not sufficient condition. The argument above simply shows that non-RKKY behaviour is possible in presence of multi-periodic $g_{n 0}$. To find out whether it really occurs one has to solve Eq.(16) and check whether there are real wave vectors $q_{j}^{s}$ for values of $m_{1}^{1}, \ldots, m_{j}^{s}, \ldots$ other than the ones obtained by the RKKY theory. 
In summary, the determination of the oscillation periods results from the analysis of the bulk spacer off-diagonal propagators where the relevant real wave vectors, under the stationary phase condition, are obtained from the band structure. The relation between the spacer FS and the oscillation periods is the same as the geometrical RKKY picture for the lowest order terms. Nevertheless, for higher order contributions a direct correspondence with the FS is not evident and the stationary phase condition displayed in Eq.(16) determines the periods with which the coupling oscillates. We have shown that oscillation periods other than the ones predicted by the RKKY theory may arise. To illustrate the method and unambiguously prove the existence and relevance of periods other than RKKY ones, we next evaluate the particular case of the FCC lattice along the (110) direction within the single-band tight-binding model.

\section{APPLYING THE METHOD}

Having presented the general method for determining the periods of the interlayer coupling, here we apply it to the particular case of a FCC(110) system within the single-band nearest-neighbour tight-binding model, which unmistakably shows the existence of nonRKKY periods. Moreover, in the light of the QWT this system is interesting because of the difficulty in determining analytically the energies of the resonances and size quantized states in such a structure [12]. For a single band the notation introduced in the previous section becomes simpler due to the omission of the band index $s$. In addition, all the matrices over orbital indices become scalars.

We label the nearest neighbour hopping $t_{0}$ and with no loss of generality assume the on-site potential $\epsilon_{0}=0$. The bulk density of states of the FCC lattice is displayed in Fig.2. It is worth pointing out that there are different contributions to the interlayer coupling, depending on the position of the Fermi energy inside the band. Three distinct regions are observed and their boundaries are shown by the vertical dashed lines in Fig.2. In addition

to the traditional polyhedral BZ, we make use of an auxiliary prismatic BZ due to the 
2-dimensional symmetry of the multilayers [5]. Both zones are equivalent and enclose the same volume in reciprocal space. The energy boundaries indicated correspond to Fermi energies such that the FS just touches the two distinct Brillouin zones. More specifically, for $E_{F}=-4 t_{0}$ the corresponding FS touches the prismatic BZ, whereas $E_{F}=0$ is the exact Fermi energy where the FS opens the necks which are familiar from the noble metals [14].

As previously mentioned, even within the nearest neighbour tight-binding model, in this case electrons are capable of hopping between nearest and next nearest planes. The bulk band structure is then given by

$$
E\left(\mathbf{q}_{\|}, q_{\perp}\right)=\epsilon\left(\mathbf{q}_{\|}\right)+2 t_{1}\left(\mathbf{q}_{\|}\right) \cos \left(q_{\perp} d\right)+2 t_{2}\left(\mathbf{q}_{\|}\right) \cos \left(2 q_{\perp} d\right) .
$$

We assume the $z$ direction perpendicular to the layers. The 2-dimensional BZ over which the stationary solutions must be searched for is a rectangle defined by $-\pi / 2 d \leq q_{x} \leq \pi / 2 d$ and $-\pi \sqrt{2} / 4 d \leq q_{y} \leq \pi \sqrt{2} / 4 d$, where $q_{x}$ and $q_{y}$ are the components of $\mathbf{q}_{\|}$. The explicit dependence of $E$ on $\mathbf{q}_{\|}$is given by $\epsilon\left(\mathbf{q}_{\|}\right)=-2 t_{0} \cos \left(2 q_{x} d\right), t_{1}\left(\mathbf{q}_{\|}\right)=-4 t_{0} \cos \left(q_{x} d\right) \cos \left(\sqrt{2} q_{y} d\right)$ and $t_{2}\left(\mathbf{q}_{\|}\right)=-t_{0}$.

Following the steps of the previous section, $\omega^{+}-E\left(\mathbf{q}_{\|}, q_{\perp}\right)=0$ gives the poles which yield the periods of $g_{n 0}$. Eq.(19) can be rewritten in terms of a second degree polynomium in $\cos \left(q_{\perp} d\right)$ whose solutions $q_{1}$ and $q_{2}$ are given by

$$
\cos \left(q_{j} d\right)=-\left\{\frac{\gamma+(-1)^{j} \sqrt{\gamma^{2}+\frac{8\left(\omega^{+}-\epsilon+2 t_{2}\right)}{2 t_{2}}}}{4}\right\},
$$

where $\gamma\left(\mathbf{q}_{\|}\right)=t_{1} / t_{2}$ and $j$ is either 1 or 2 . Nevertheless, the poles $q_{1}$ and $q_{2}$ are in the $q_{\perp}$-complex plane. By mapping the relationship between the two complex functions we can uniquely relate one value of $q_{\perp}$ inside the integration contour to the corresponding value

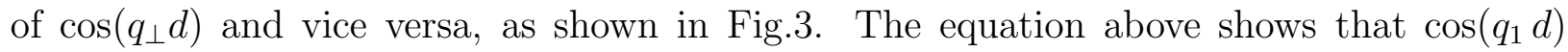
and $\cos \left(q_{2} d\right)$ have opposite imaginary parts and therefore, following the maps in Fig. 包, $q_{1}$ and $q_{2}$ have opposite real parts. This confirms what has been said in the previous section concerning the position of two different wave vectors for a given band $s$.

In addition, looking at Eq.(20) with $\omega=E_{F}$ it is clear that non-RKKY behaviour cannot arise in the bottom region of the band $\left(-12 t_{0}<E_{F}<-4 t_{0}\right)$. This is due to the fact that 
in such a region $q_{1}$ and $q_{2}$ cannot both be real. In fact, the corresponding FS in this region is spherical-like. However, in the range $-4 t_{0}<E_{F}<4 t_{0}$, corresponding to the top regions inside the band, both $q_{1}$ and $q_{2}$ may be real indicating that non-RKKY periods are possible.

Having obtained the poles, from Eqs.(9) and (10) we have

$$
g_{n 0}\left(\mathbf{q}_{\|}, \omega\right)=A_{1}\left(\mathbf{q}_{\|}, \omega\right) e^{i q_{1}\left(\mathbf{q}_{\|}, \omega\right) n d}+A_{2}\left(\mathbf{q}_{\|}, \omega\right) e^{i q_{2}\left(\mathbf{q}_{\|}, \omega\right) n d},
$$

where

$$
A_{j}\left(\mathbf{q}_{\|}, \omega\right)=\left\{2 i\left[\cos \left(q_{1} d\right)-\cos \left(q_{2} d\right)\right] \sqrt{1-\cos ^{2}\left(q_{j} d\right)}\right\}^{-1}
$$

In the present case $g_{0 n}=g_{n 0}$. Eq.(21) shows that for values of $\mathbf{q}_{\|}$and $\omega$, for which $q_{1}$ and $q_{2}$ are real, $g_{n 0}$ oscillates with the superposition of two periods, $2 \pi d /\left|q_{1}\right|$ and $2 \pi d /\left|q_{2}\right|$, which are in general incommensurate. In those cases $g_{n 0}$ exhibits a quasi-periodic dependence on $n$.

Because there are only two poles in this case, the function $\mathcal{F}$, following Eq.(12), becomes

$$
\mathcal{F}=\sum_{m_{1}, m_{2}} C_{m_{1}, m_{2}} e^{i\left(m_{1} q_{1}+m_{2} q_{2}\right) n d}
$$

where the Fourier coefficients are given by Eq.(13). Within the single-band model, the Fourier coefficients can be calculated in an alternative way, shown in Appendix A. Following the Appendix, we recall that the lowest order Fourier coefficients correspond to the RKKYlimit $\mathcal{F}_{R K K Y}$. They are $C_{m_{1}, m_{2}}$ where $m_{1}+m_{2}=2,\left|m_{1}\right| \leq 2$ and $\left|m_{2}\right| \leq 2$. More specifically, $C_{2,0}=\frac{\mathcal{T} A_{1}^{2}}{2 i}, C_{0,2}=\frac{\mathcal{T} A_{2}^{2}}{2 i}, C_{1,1}=\frac{\mathcal{T} A_{1} A_{2}}{i}$, and the property $C_{-m_{1},-m_{2}}=\left(C_{m_{1}, m_{2}}\right)^{*}$ define all the six coefficients present in the RKKY expression, where $\mathcal{T}=4 \tau_{\uparrow} \tau_{\downarrow}\left(1-\tau_{\uparrow} g_{00}\right)\left(1-\tau_{\downarrow} g_{00}\right)$ and $\tau_{\uparrow \downarrow}$ are defined after Eq.(四). Beyond the lowest order coefficients are the terms expected to contribute to the non-RKKY behaviour. It is then illustrative to present one of these terms, namely $C_{3,1}$,

$$
C_{3,1}=\frac{2 \mathcal{T} A_{1}^{3} A_{2}}{i}\left[\left(\tau_{\uparrow}\right)^{2}+\left(\tau_{\downarrow}\right)^{2}-\frac{\mathcal{T}}{2}\right]
$$

The stationary phase condition in Eq.(16) is rewritten as 


$$
m_{1} \nabla_{\|} q_{1}\left(\mathbf{q}_{\|}, E_{F}\right)+m_{2} \nabla_{\|} q_{2}\left(\mathbf{q}_{\|}, E_{F}\right)=0
$$

The condition above imposes that both gradients must be colinear and moreover, the ratio between their moduli must be a rational number. The determination of the stationary points $\mathbf{q}_{\|}^{\alpha}=\left(q_{x}, q_{y}\right)$ yielding the periods $\lambda_{m_{1}, m_{2}}^{\alpha}=2 \pi /\left|m_{1} q_{1}^{\alpha}+m_{2} q_{2}^{\alpha}\right|$ is rather tedious but straightforward. As previously mentioned, the solutions are often located at highly symmetric points of the BZ but more general solutions may occur. Nevertheless, because the RKKY periods as well as the most important higher order terms come only from those high-symmetry points, we shall firstly focus on these solutions. Across the whole energy band, four stationary points are found on the corners of the 2-dimensional irreducible BZ. They are $\mathbf{q}_{\|}^{a}=(0,0), \mathbf{q}_{\|}^{b}=(0, \pm \pi \sqrt{2} / 4 d), \mathbf{q}_{\|}^{c}=( \pm \pi / 2 d, \pm \pi \sqrt{2} / 4 d)$ and $\mathbf{q}_{\|}^{d}=( \pm \pi / 2 d, 0)$.

In the first region $\lambda_{2,0}^{a}$ is the only period, which is actually present along the entire band. The graphical relation between the spacer FS and the oscillation period is displayed in Fig. 4 , where the FS for an arbitrary energy $\left(E_{F} / 2 t_{0}=-3.0\right)$ is shown together with the wave vector $q_{1}^{a}$ and the cross section of the prismatic BZ for $q_{y}=0$. The cross section is defined by $-\pi / 2 d \leq q_{x} \leq \pi / 2 d$ and $-\pi / d \leq q_{z} \leq \pi / d$. Note that $2 \mathbf{q}_{1}^{a}$ corresponds to the extremal wave vector spanning the FS, in according to the RKKY picture. Clearly, higher order periods $\lambda_{m_{1}, 0}$ for $m_{1}>2$ are merely harmonics of the fundamental $\lambda_{2,0}$.

In the second region an additional period $\lambda_{1,1}^{b}$ arises. Similarly, the vector $q_{1}^{b}+q_{2}^{b}$ yields the fundamental period and possible higher combinations $m_{1}\left(q_{1}^{b}+q_{2}^{b}\right)$ add no new terms but harmonics. Fig. 5 shows a cross section of the FS $\left(E_{F} / 2 t_{0}=-1.0\right)$ for $q_{y}=\pi \sqrt{2} / 4 d$. The wave vectors $\mathbf{q}_{1}^{b}$ and $\mathbf{q}_{2}^{b}$ are highlighted and it is clear that $\mathbf{q}_{1}^{b}+\mathbf{q}_{2}^{b}$ satisfies the RKKY criteria. At first glance one could think, accordingly to Fig.5, that $2 \mathbf{q}_{1}^{b}$ and $2 \mathbf{q}_{2}^{b}$ are also contributory wave vectors for the same reasons as in the first region. Nevertheless, by looking at the $q_{y}$-dependence of the FS we find that those vectors are not solutions of Eq.(25).

The most interesting case occurs in the top region, where the FS has necks resembling those of the noble metals. In addition to $\lambda_{2,0}^{a}$ at $\mathbf{q}_{\|}^{a}$, other stationary solutions occur at $\mathbf{q}_{\|}^{c}$ and $\mathbf{q}_{\|}^{d}$. At $\mathbf{q}_{\|}^{d}$, analogously to the second region, the solution comes from $\mathbf{q}_{1}^{d}+\mathbf{q}_{2}^{d}$ and does 
not introduce new periods. At $\mathbf{q}_{\|}^{c}$ though, both gradients in Eq.(25) vanish simultaneously allowing any values of $m_{1}$ and $m_{2}$ to satisfy the stationary phase condition. Therefore, the coupling oscillates with as many periods $\lambda_{m_{1}, m_{2}}^{c}$ as there are non-zero coefficients $C_{m_{1}, m_{2}}^{c}$. The lowest order $\lambda_{2,0}^{c}, \lambda_{0,2}^{c}$ and $\lambda_{1,1}^{c}$ correspond to the RKKY periods and the geometrical relation between the wave vectors $\mathbf{q}_{1}^{c}, \mathbf{q}_{2}^{c}$ and the FS in Fig.6 confirms this point. For the present model we find that $q_{1}^{c}-q_{2}^{c}=\pi$. Thus, the oscillation periods $\lambda_{2,0}^{c}$ and $\lambda_{0,2}^{c}$ cannot be distinguished just by looking at discrete integer values of $n$. In addition, $\lambda_{1,1}^{c}=\lambda_{1,1}^{d}$, which leads the third energy region to contain three non-equivalent RKKY periods. We recall that in $\mathrm{FCC} \mathrm{Cu} E_{F}$ lies in the third energy region with FS necks. A quantitative description of the oscillation periods for $\mathrm{Cu}$ can be obtained within the present framework by going beyond nearest neighbours and using the tight-binding parameters of Halse [13]. Then $\lambda_{1,1}^{c}$ and $\lambda_{1,1}^{d}$ become distinct periods and, by taking into account the equivalence of $\lambda_{2,0}^{c}$ and $\lambda_{0,2}^{c}$ for a discrete lattice, we find exactly the four RKKY periods of Bruno and Chappert 《ᄈ.

However, because in general the wave vectors $\mathbf{q}_{1}^{c}$ and $\mathbf{q}_{2}^{c}$ are incommensurate, higher order periods are not simple harmonics of the RKKY ones, unlike in the two lower regions. In fact, the period $\lambda_{3,1}^{c}$ does not correspond to any harmonics of the fundamental RKKY periods. Furthermore, even though the wave vectors $\mathbf{q}_{1}^{c}$ and $\mathbf{q}_{2}^{c}$ separately have clear correspondences with the FS, as shown in Fig.6, the wave vector $3 \mathbf{q}_{1}^{c}+\mathbf{q}_{2}^{c}$ has not. From this argument it becomes clear that higher order periods do not satisfy the geometrical picture of the RKKY theory, having instead an indirect relation between the wave vectors and the FS.

Fig.7] summarizes the results obtained here by displaying the oscillation periods as a function of the Fermi energy $E_{F}$. The full lines labeled from 1 to 5 are the fundamental RKKY periods $\lambda_{2,0}^{a}, \lambda_{1,1}^{b}, \lambda_{2,0}^{c}, \lambda_{0,2}^{c}$ and $\lambda_{1,1}^{c}$, respectively, and the dot-dashed line in the top region represents only one of the non-RKKY periods, namely $\lambda_{3,1}^{c}$. Recalling the relation $q_{1}^{c}-q_{2}^{c}=\pi$, we see that the oscillation periods $\lambda_{2,0}^{c}$ and $\lambda_{0,2}^{c}$ cannot be distinguished just by looking at discrete integer values of $n$. Still focusing on the top energy region, Fig.8 exhibits the bilinear coupling $J_{1}=\Delta \Omega(\pi) / 2$ at temperature $T=2.0 \times 10^{-3} W / k_{B}$ as a function of the spacer thickness for $E_{F} / 2 t_{0}=1.64$ and $V_{e x}=0.15 W$, where $W=16 t_{0}$ is the spacer band- 
width. This value of $E_{F}$ is chosen so that $\lambda_{3,1}^{c}$ is well separated from the RKKY periods, as may be seen in Fig.7. The full line in Fig.8 corresponds to the result obtained from Eqs.(11) and (2), whereas the dashed line is the RKKY approximation, following Eqs.(11) and (5), scaled down by a factor of 8 for reasons of comparison. Despite the agreement in the dominant long period, the difference in the fine structure of the oscillations reflects contributions beyond the fundamental RKKY periods.

The evaluation of the stationary phase approximation in this case unmistakably proves the existence and relevance of the non-RKKY periods. Fig.9 displays the absolute value of the ratio between some Fourier coefficients $C_{m_{1}, m_{2}}^{c}$ and $C_{1,1}^{c}$, the largest coefficient of a fundamental RKKY period, as a function of $V_{e x}$. The full line corresponds to the Fourier coefficient associated with the new period $\lambda_{3,1}^{c}$ whereas the dashed and dot-dashed lines correspond to $\lambda_{4,2}^{c}$ and $\lambda_{4,0}^{c}$, respectively, which are simple harmonics of the fundamental RKKY periods. As expected for very small exchange splittings, the magnitudes of higher order coefficients relative to that of the fundamental RKKY ones are negligible [6], indicating that non-RKKY behaviour becomes more important as the exchange splitting is increased. An interesting consequence is that, even though the periods are determined from the spacer FS, their relevances depend on the nature of the magnetic materials involved. Note that for $V_{e x} \approx 0.15 W$ the ratio $\left|C_{3,1}^{c}\right| /\left|C_{1,1}^{c}\right| \approx 1 / 2$. This indicates that for such a value of $V_{e x}$, which is exactly the same as in Fig. 8, the Fourier coefficients are of the same order of magnitude; the only reason why both periods do not have comparable weights is due to geometrical factors determined by the curvatures of the respective surfaces. In fact, the curvature of the surface $q_{1}+q_{2}$ vanishes at $\mathbf{q}_{\|}^{c}$, making it impossible to evaluate the stationary phase approximation for the period $\lambda_{1,1}^{c}$ even though it undoubtedly indicates a strong contribution. Taking into account the geometrical curvatures when possible, we evaluate the stationary phase approximation and show in Fig. 10 the separate contribution of some periods to the exchange coupling. The full line is the new period $\lambda_{3,1}^{c}$ whereas the dashed line and the the dot-dashed line represent $\lambda_{2,0}^{a}$ and $\lambda_{2,0}^{c}$, respectively. Clearly, $\lambda_{3,1}^{c}$ is as important as the RKKY periods, except for the long dominant one. It is worth pointing 
out that the rate of decay of the oscillation amplitude associated with the new periods is exactly the same as for the lowest order ones, falling off as $1 / n^{2}$ for $T=0$. Concerning the actual amplitude of the oscillations, two points should be highlighted. The strength of all periods depends rather critically on the matching of the bands in real materials, which is not well reproduced by our single-orbital model, and the omission of the energy dependence of the Fourier coefficients could be serious for Cu-based systems [16].

The solutions treated above all come from the high-symmetry points $\mathbf{q}_{\|}^{a}, \mathbf{q}_{\|}^{b}$, $\mathbf{q}_{\|}^{c}$ and $\mathbf{q}_{\|}^{d}$. Note that fundamentally new periods arise but always in the presence of RKKY ones. They result from crossed mixtures of the fundamental wave vectors, which in turn yield the RKKY periods. Nevertheless in general, as previously stated, solutions of Eq.(25) away from high-symmetry points are found and we point out that their contributions are not necessarily associated with the existence of RKKY periods. In fact, it can be shown in the second energy region that energy-dependent solutions exist along the line $q_{x}=0$ with corresponding wave vectors $\mathbf{q}_{1}$ and $\mathbf{q}_{2}$ which do not yield any possible RKKY period. This indicates there are colinear gradients along that line satisfying Eq.(25) for different values of $m_{1}$ and $m_{2}$ even though no RKKY stationary solutions are obtained. These solutions are found to be far less significant in their contribution to the exchange coupling than the highly symmetric ones.

\section{CONCLUSIONS}

In summary, we have shown that the RKKY theory is not capable of predicting all the oscillation periods of the interlayer coupling, as generally believed. The actual relation between the periods and the spacer FS is more subtle than the RKKY geometrical picture. Even though the lowest order periods indeed correspond to the RKKY predictions, nonnegligible higher order terms arise and do not bear any direct correspondence to the FS. Instead, they correspond to indirect combinations of fundamental wave vectors, which in

turn, may or may not yield RKKY periods. The appearance of non-RKKY oscillations 
clearly requires the multi-periodicity of the bulk spacer off-diagonal propagator as a function of the spacer thickness, although this is not a sufficient condition. In addition, real solutions of the stationary phase condition must exist in order to have a constructive interference between the electrons across the system. The otherwise destructive interference damps the oscillation not contributing to the coupling. Finally, if such behaviour can be found even within the single-band model, for which the FS is simple and has a single sheet, we may expect the occurrence of non-RKKY periods in systems with more elaborate FS. Surfaces with more than one sheet, such as non-magnetic transition metals for instance, are possible candidates for presenting such periods. In those cases, the interpretation of the results in terms of the RKKY theory may be misleading.

We are grateful to EPSRC and Royal Society of UK, and CNPq of Brazil for financial support. We also would like to acknowledge useful discussions with Dr. R. B. Muniz and Dr. A. Umerski.

\section{APPENDIX A}

Alternatively, within the single-band model, the Fourier coefficients $C_{m_{1}, m_{2}}$ in section 【II can be calculated in the following way. Bearing in mind the equivalence between $g_{0 n}=g_{n 0}$, Eq.(㺼) can be concisely written as

$$
G_{n 0}^{\uparrow}=\frac{\tau_{\uparrow} g_{0 n}\left(1-\tau_{\uparrow} g_{00}\right)}{V_{e x}} \sum_{j=0}^{\infty}\left(\tau_{\uparrow} g_{n 0}\right)^{2 j}
$$

and analogously,

$$
G_{0 n}^{\downarrow}=-\frac{\tau_{\downarrow} g_{0 n}\left(1-\tau_{\downarrow} g_{00}\right)}{V_{e x}} \sum_{j^{\prime}=0}^{\infty}\left(\tau_{\downarrow} g_{n 0}\right)^{2 j^{\prime}} .
$$

The product of Eqs.(A1) and (A2) is

$$
G_{n 0}^{\uparrow} G_{0 n}^{\downarrow}=-\frac{\tau_{\uparrow} \tau_{\downarrow}\left(1-\tau_{\uparrow} g_{00}\right)\left(1-\tau_{\downarrow} g_{00}\right)}{\left(V_{e x}\right)^{2}} \sum_{j, j^{\prime}}\left(\tau_{\uparrow}\right)^{2 j}\left(\tau_{\downarrow}\right)^{2 j^{\prime}}\left(g_{n 0}\right)^{2\left(j+j^{\prime}+1\right)} .
$$

Writing the logarithmic function as a series, we have from Eq.(2) 


$$
\mathcal{F}=\operatorname{Im}\left\{\sum_{\ell=1}^{\infty} \frac{(-1)^{\ell+1}}{\ell}\left[\mathcal{T} \sum_{j, j^{\prime}}\left(\tau_{\uparrow}\right)^{2 j}\left(\tau_{\downarrow}\right)^{2 j^{\prime}}\left(g_{n 0}^{2}\right)^{\left(j+j^{\prime}+1\right)}\right]^{\ell}\right\}
$$

where $\mathcal{T}=-4 \tau_{\uparrow} \tau_{\downarrow}\left(1-\tau_{\uparrow} g_{00}\right)\left(1-\tau_{\downarrow} g_{00}\right)$. We recall that the summations above have indices with different limits. Whereas the external summation index $\ell$ runs over the integers starting from $\ell=1$ onwards, the inner indices $j$ and $j^{\prime}$ have zero as their initial values. Eq.(A4) can be manipulated and rewritten as

$$
\mathcal{F}=\operatorname{Im}\left\{\sum_{\ell=1}^{\infty} \frac{(-1)^{\ell+1}}{\ell} \mathcal{T}^{\ell} \sum_{j_{1}, j_{1}^{\prime}} \ldots \sum_{j_{\ell}, j_{\ell}^{\prime}}\left(\tau_{\uparrow}\right)^{2\left(j_{1}+\ldots+j_{\ell}\right)}\left(\tau_{\downarrow}\right)^{2\left(j_{1}^{\prime}+\ldots+j_{\ell}^{\prime}\right)}\left(g_{n 0}^{2}\right)^{\left(j_{1}+\ldots+j_{\ell}+j_{1}^{\prime}+\ldots+j_{\ell}^{\prime}+\ell\right)}\right\} .
$$

For a fixed $\ell$ there are twice as many inner summations, running from $j_{1}$ and $j_{1}^{\prime}$ as far as $j_{\ell}$ and $j_{\ell}^{\prime}$. We define the integer $p$ as the exponent of the function $g_{n 0}^{2}$ in Eq.(A5), i.e., $p=j_{1}+j_{1}^{\prime}+\ldots+j_{\ell}+j_{\ell}^{\prime}$. Note that $p$ runs over the positive integers greater than zero.

Squaring $g_{n 0}$ in Eq.(21) and inserting it into Eq.(A5) we obtain the function $\mathcal{F}$ in terms of a Fourier expansion. Clearly, the exponential $e^{i\left(m_{1} q_{1}+m_{2} q_{2}\right) n d}$ arises from terms of $\left(g_{n 0}\right)^{2 p}$ such that $p=\frac{m_{1}+m_{2}}{2}$. The number of terms in turn depends on the number of possible combinations between the implicit indices $j, j^{\prime}$ and $\ell$, and the larger $p$ is, the more combinations are possible.

For $p=1$ for instance, the only possible combination of the indices is $j_{1}=j_{1}^{\prime}=0$ and $\ell=1$. The terms coming from this contribution correspond to the RKKY-limit expression in Eq.(5), which indicates $\mathcal{F}_{R K K Y}$ as a subset of the full expression $\mathcal{F}$ and confirms the RKKY theory as a limit of the more general approach used here [6].

For $p=2$, more combinations are possible. In addition to $\ell=2$ and $j_{1}=j_{1}^{\prime}=j_{2}=$ $j_{2}^{\prime}=0$, four other combinations come from $\ell=1$ and $j_{1}+j_{1}^{\prime}+j_{2}+j_{2}^{\prime}=1$. Note that the lowest order coefficients are easily calculated through this alternative method but as the exponent $p$ is increased, more terms arise and increase the difficulty. Nevertheless, because we are searching for periods other than the RKKY ones, the lowest order terms beyond the RKKY-limit are expected to be the important corrections to the coupling. 
Finally, from the discussion above, two properties of the Fourier coefficients are straightforwardly obtained. They are $C_{-m_{1},-m_{2}}=C_{m_{1}, m_{2}}^{*}$ and $C_{m_{1}, m_{2}}=0$ unless $m_{1}+m_{2}$ is even.

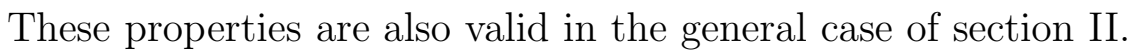




\section{REFERENCES}

[1] D. M. Edwards, J. Mathon, R. B. Muniz and M. S. Phan, Phys. Rev. Lett. 67, 493 (1991).

[2] D. M. Edwards, J. Mathon, R. B. Muniz and M. S. Phan, J. Phys.: Condens. Matter 3 $4941(1991)$

[3] J. Mathon, Murielle Villeret and D. M. Edwards, J. Phys.: Condens. Matter 4, 9873 (1992)

[4] P. Bruno and C. Chappert, Phys. Rev. Lett. 67, 1602 (1991)

[5] P. Bruno and C. Chappert, Phys. Rev. B 46, 261 (1992)

[6] J. d'Albuquerque e Castro, M. S. Ferreira and R. B. Muniz, Phys. Rev. B 49, 16062 (1994)

[7] M. S. Ferreira, J. d'Albuquerque e Castro, D. M. Edwards and J. Mathon, J.M.M.M. 154, L1 (1996)

[8] J. d'Albuquerque e Castro, J. Mathon, Murielle Villeret and D. M. Edwards, Phys. Rev. B 51, $12876(1995)$

[9] M. S. Ferreira, J. d'Albuquerque e Castro, R. B. Muniz and D. M. Edwards, J. Phys.: Condens. Matter 6, L619 (1994)

[10] D. M. Edwards, J. Mathon and R. B. Muniz, Phys. Rev. B 5016066 (1994)

[11] B. Heinrich et al., Phys. Rev. B 44, 9348 (1991)

[12] A. V. Kozlov and W. A. Harrison, Phys. Rev. B 4812334 (1993)

[13] M. R. Halse, Trans. Roy. Soc. London A 265, 507 (1969)

[14] N. W. Ashcroft and N. D. Mermin, Solid State Physics (1976)

[15] D. M. Edwards, A.M. Robinson and J. Mathon, J.M.M.M. 140-144, 157 (1995) 
[16] J. d'Albuquerque e Castro, J. Mathon, Murielle Villeret and A. Umerski, Phys. Rev. B 53, $13306(1996)$

[17] P. Bruno, Phys. Rev. B 52, 411 (1995) 


\section{FIGURES}

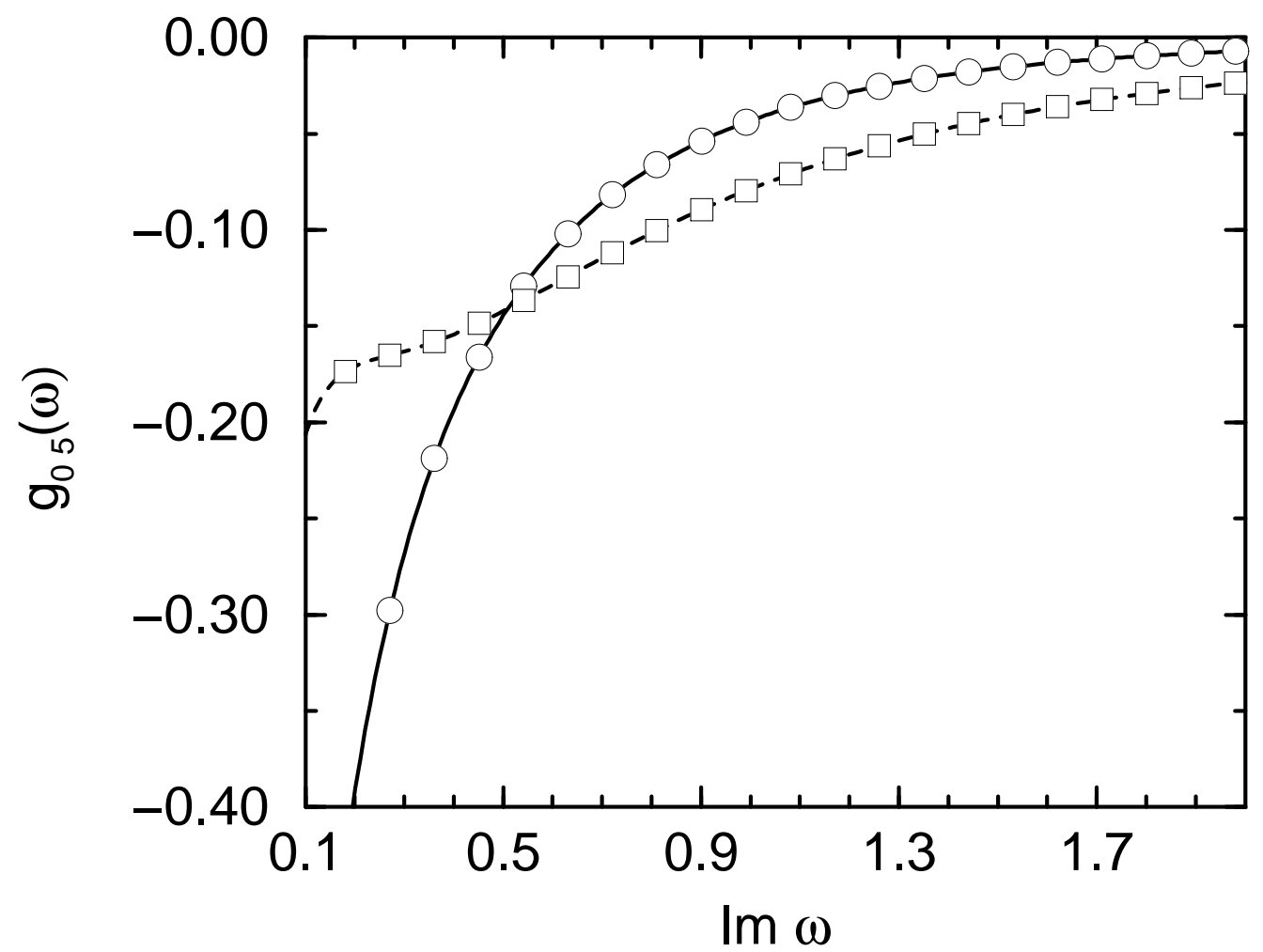

FIG. 1. Comparison between the analytical expression and the brute-force calculation of the propagator $g_{05}(\omega)$. The lines represent the analytical results and the symbols the numerical ones. The full line and the circles are the real part of $g_{05}$ whereas the dashed line and the squares are the imaginary part. The parameters of this tight-binding Hamiltonian are indicated in the text. In order to improve the convergence of the brute-force calculations we have varied $\omega$ along the imaginary axis. 


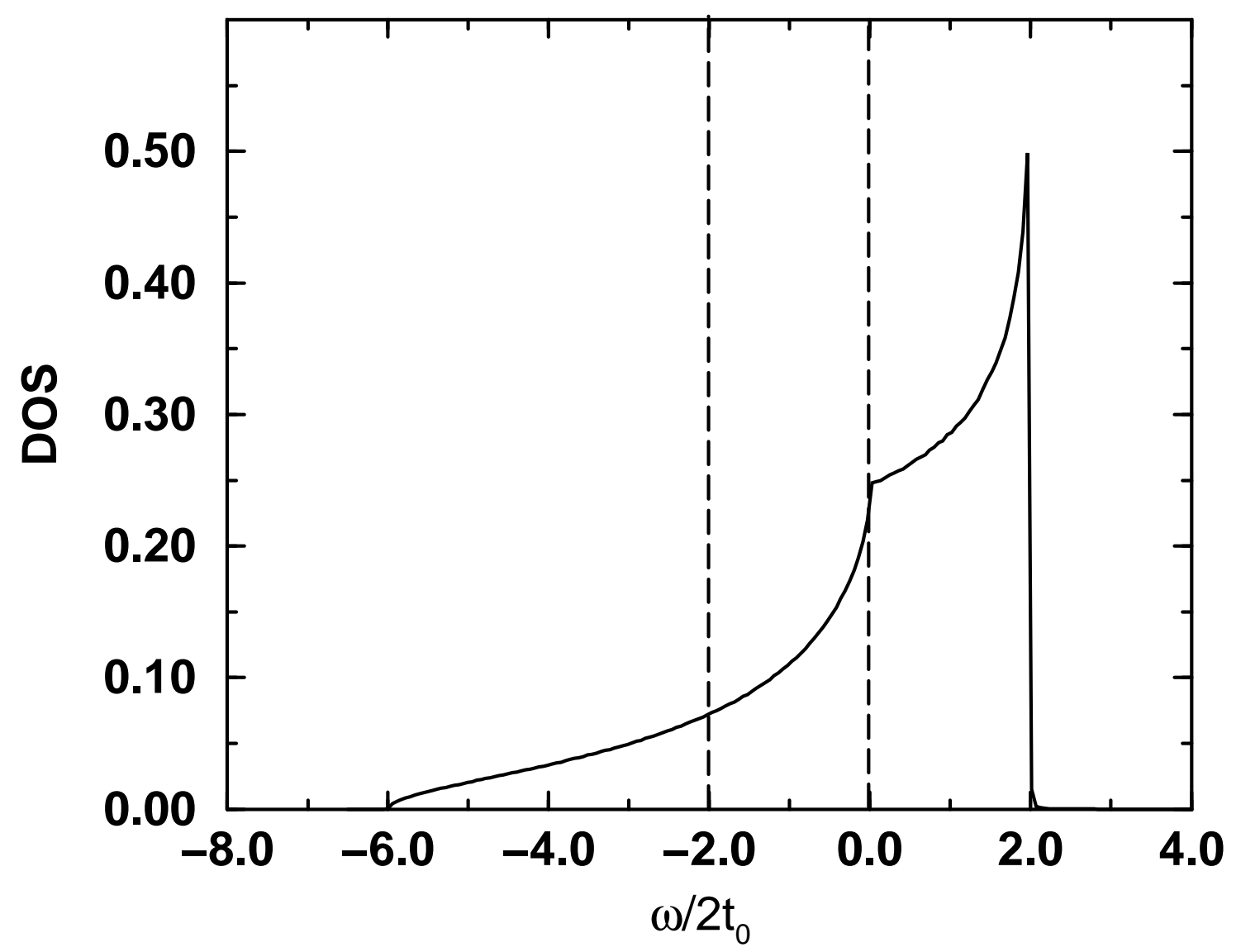

FIG. 2. Density of states of the bulk FCC tight-binding model. The vertical dashed lines separate the three distinct regions. 

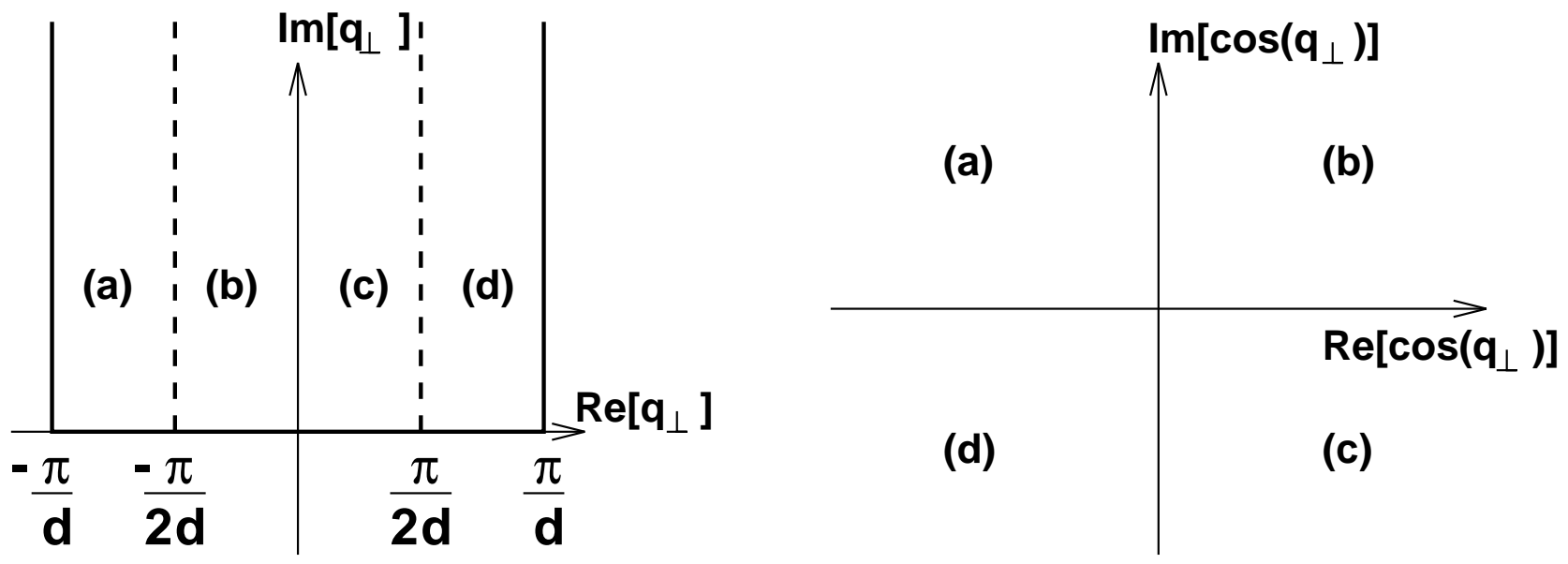

FIG. 3. Correspondence between the $q_{\perp}$ and $\cos \left(q_{\perp}\right)$-complex planes for $\ell<m$. By considering $q_{\perp}$ inside the integration contour (bold path on the left), each point on one plane has an unique correspondence on the other one. For the case $\ell>m$, since $\cos \left(q_{\perp}\right)$ is even, the map of the $q_{\perp}$-complex plane must be reflected about the axes. 


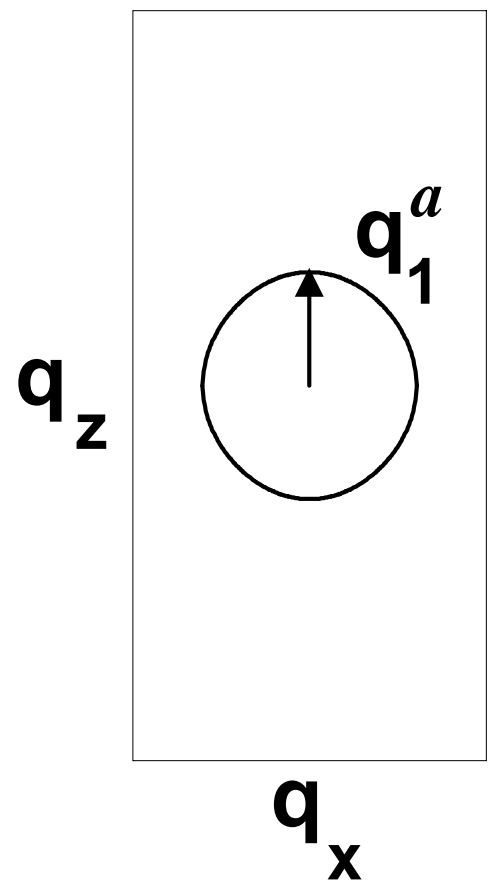

FIG. 4. Cross section of the Fermi surface for $E_{F}=-3.0$ (Region 1) and $q_{y}=0$. The rectangle is a cross section of the prismatic Brillouin zone and the wave vector $q_{1}^{a}$ is also displayed. 


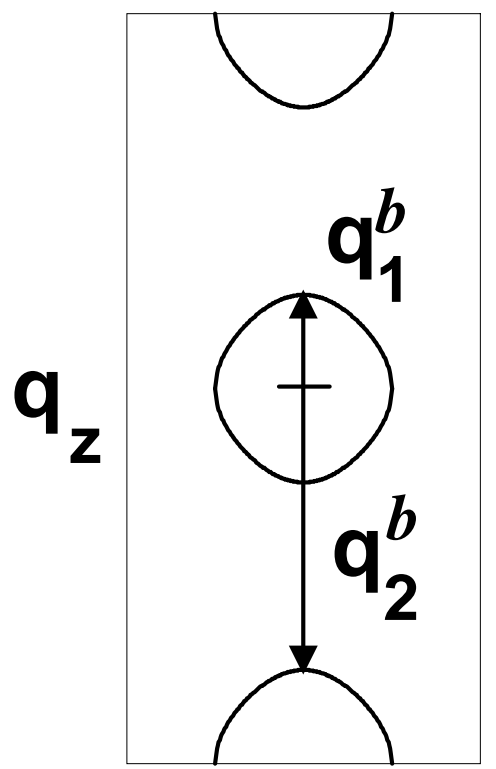

$q_{x}$

FIG. 5. Cross section of the Fermi surface for $E_{F}=-1.0$ (Region 2) and $q_{y}=\pi \sqrt{2} / 4 d$. The rectangle is a cross section of the prismatic Brillouin zone. The wave vectors $q_{1}^{b}$ and $q_{2}^{b}$ are also displayed. 


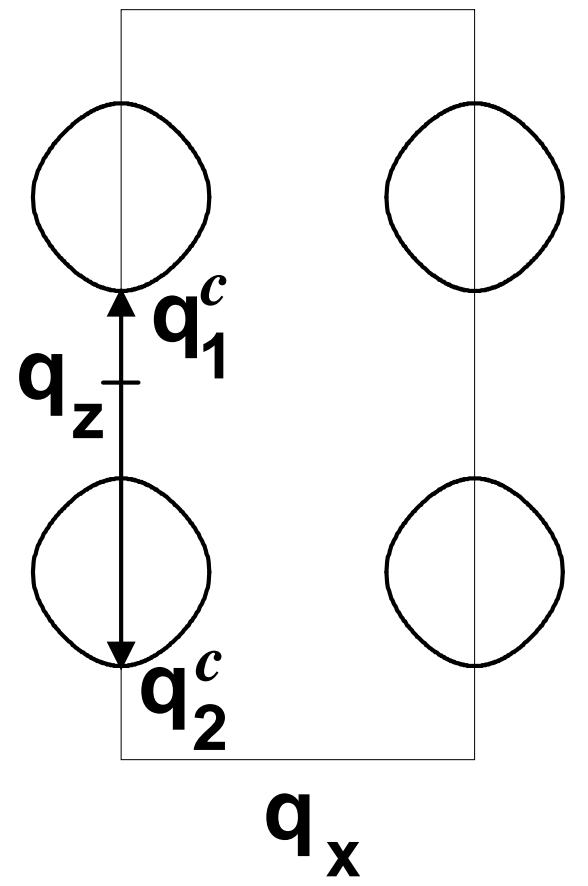

FIG. 6. Cross section of the Fermi surface for $E_{F}=1.0$ (Region 3 ) and $q_{y}=\pi \sqrt{2} / 4 d$. The rectangle is a cross section of the prismatic Brillouin zone. The wave vectors $q_{1}^{c}$ and $q_{2}^{c}$ are also displayed. 


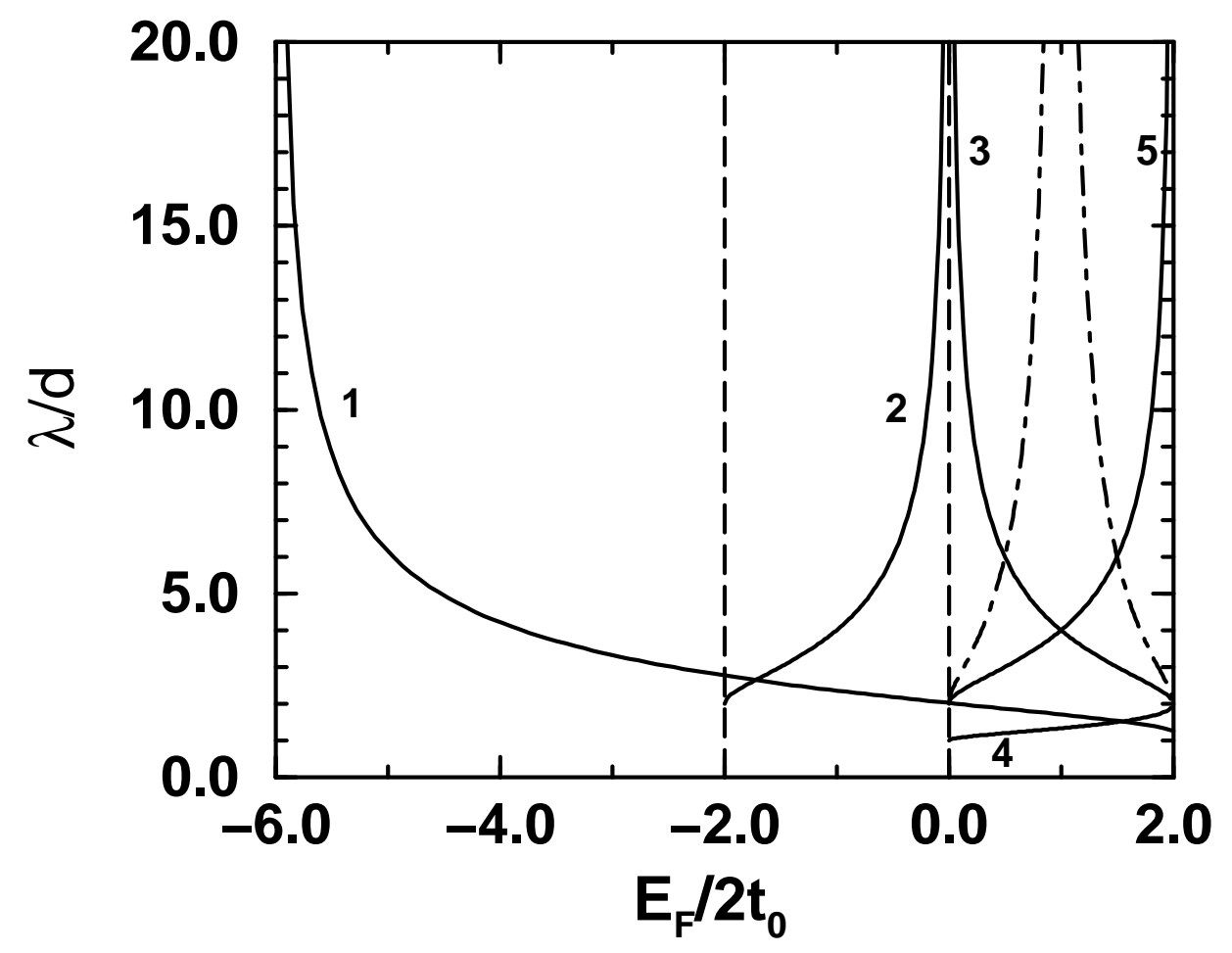

FIG. 7. Oscillation periods of the interlayer coupling as a function of the Fermi energy $E_{F}$. The vertical dashed lines separate the three distinct regions inside the band. The full lines labeled from 1 to 5 are the RKKY periods (see text) whereas the dot-dashed line in the top region is one of the non-RKKY periods, namely $\lambda_{3,1}^{c}$. 


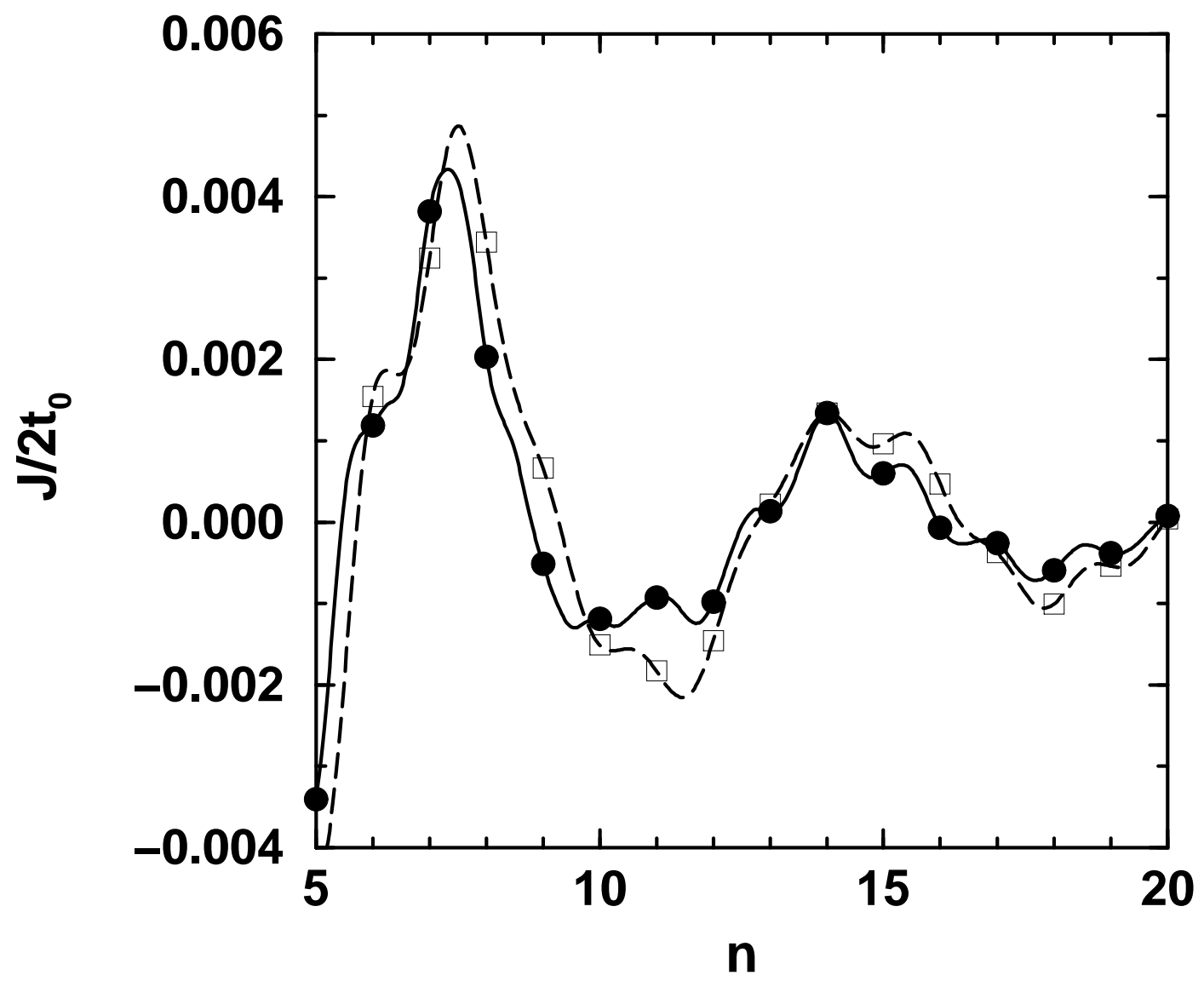

FIG. 8. Full line shows the interlayer coupling for $E_{F} / 2 t_{0}=1.64, V_{e x}=0.15 \mathrm{~W}$ and $k_{B} T=2.0 \times 10^{-3} \mathrm{~W}$, whereas the dashed line is the RKKY prediction for the same parameters scaled down by a factor of 8 for comparison. 


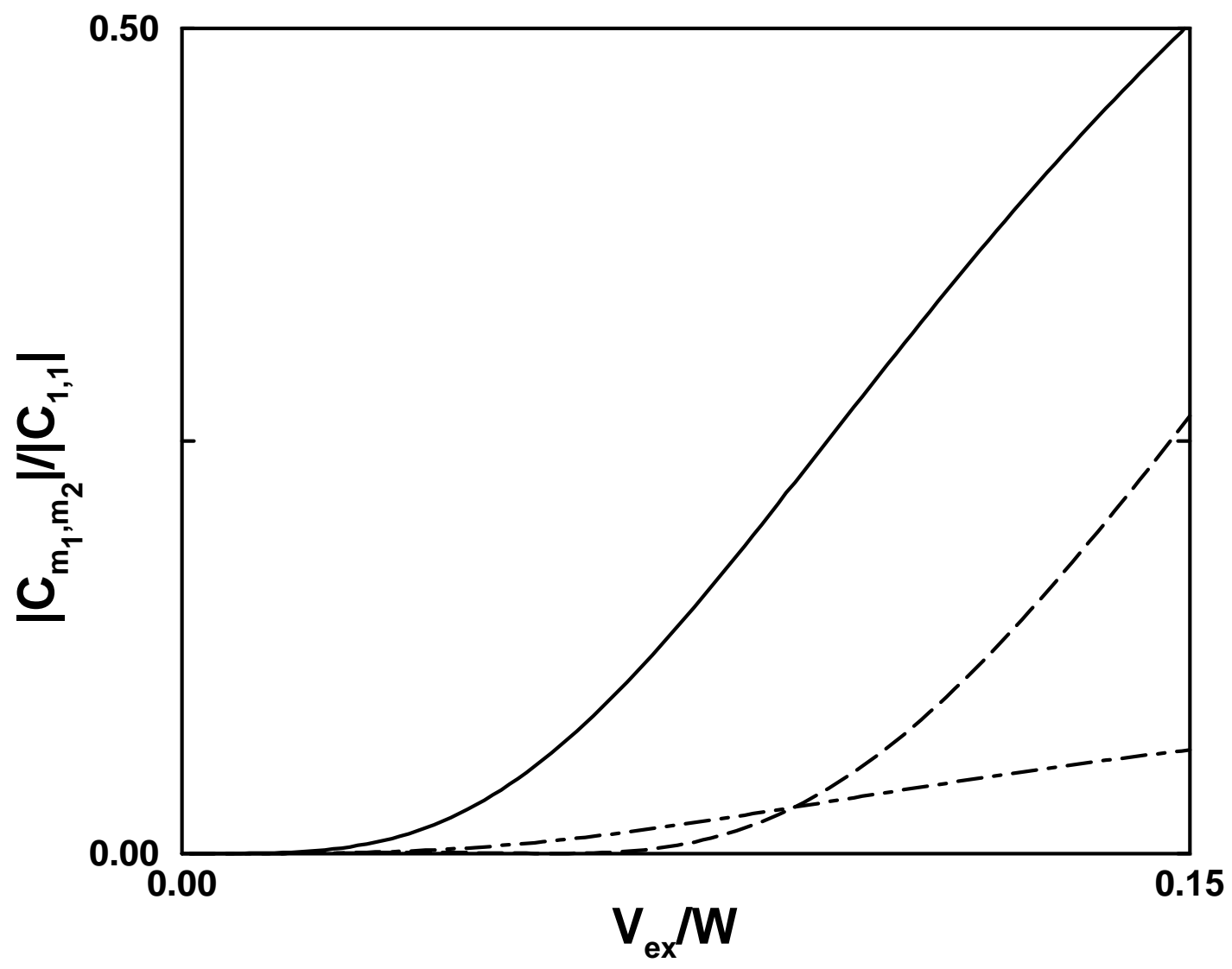

FIG. 9. Ratios $\left|C_{3,1}^{c}\right| /\left|C_{1,1}^{c}\right|$ (full line), $\left|C_{4,2}^{c}\right| /\left|C_{1,1}^{c}\right|$ (dashed line) and $\left|C_{4,0}^{c}\right| /\left|C_{1,1}^{c}\right|$ (dot-dashed line) as a function of $V_{e x}$. 


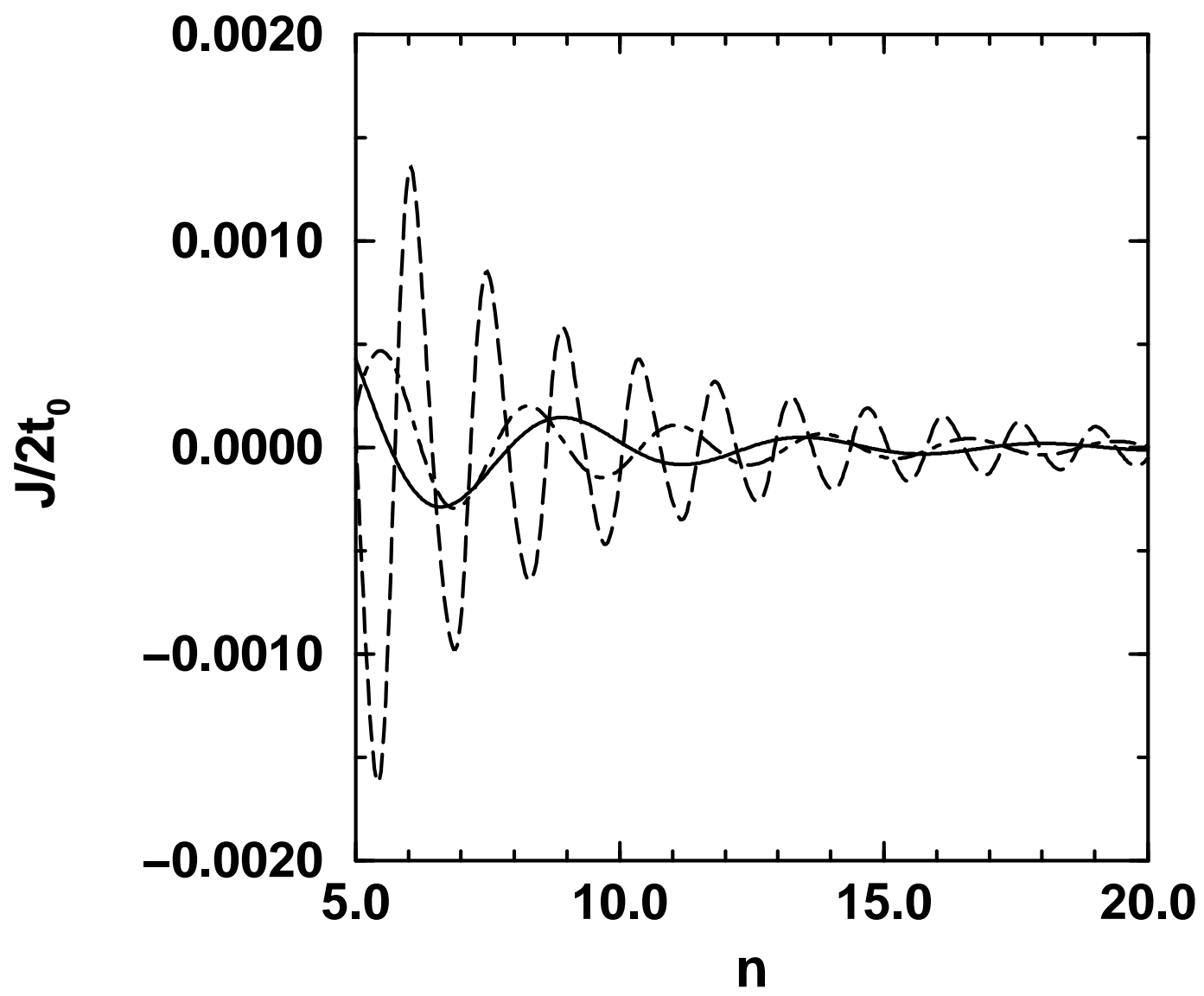

FIG. 10. Separate contributions to the interlayer coupling through the stationary phase approximation. The full line is $\lambda_{3,1}^{c}$, dashed line is $\lambda_{2,0}^{a}$ and dot-dashed line is $\lambda_{2,0}^{c} . E_{F} / 2 t_{0}=1.64$, $V_{e x}=0.15 W$ and $k_{b} T=2.0 \times 10^{-3} W$. 